\title{
Strongly Cancellative and Recovering Sets on Lattices
}

\author{
Hoda Bidkhori \\ Department of Mathematics \\ Northwestern University, Illinois, USA \\ hbidkho@ncsu.edu
}

\author{
ShinnYih Huang \\ Department of Mathematics \\ Yale University, Connecticut, USA \\ shinnyih.huang@yale.edu
}

Submitted: Dec 28, 2010; Accepted: Mar 24, 2011; Published: Mar 31, 2011

\begin{abstract}
We use information theory to study recovering sets $\mathbf{R}_{L}$ and strongly cancellative sets $\mathbf{C}_{L}$ on different lattices. These sets are special classes of recovering pairs and cancellative sets previously discussed in the papers of Simonyi, Frankl, and Füredi. We mainly focus on the lattices $B_{n}$ and $D_{l}^{k}$. Specifically, we find upper bounds and constructions for the sets $\mathbf{R}_{B_{n}}, \mathbf{C}_{B_{n}}$, and $\mathbf{C}_{D_{l}^{k}}$.
\end{abstract}

\section{Introduction}

In this paper, we study the strongly cancellative sets $\mathbf{C}_{L}$ and recovering sets $\mathbf{R}_{L}$ that are subsets of points in lattices $L$, see Definition 2.1 and 2.2. On one hand, the study of the former set is motivated by the work of Ahlswede, Frankl, and Füredi [8] and Fredman [7]. Specifically, strongly cancellative sets are a special class of cancellative sets. On the other hand, the study of recovering sets is prompted by the previous work of Simonyi [9] on recovering pairs. A recovering pair $(A, B)$ is an ordered pair of subsets $A, B$ of points in a lattice such that for any $a, a^{\prime} \in A$ and $b, b^{\prime} \in B$, we have the following:

$$
\begin{aligned}
& a \wedge b=a^{\prime} \wedge b^{\prime} \Rightarrow a=a^{\prime}, \\
& a \vee b=a^{\prime} \vee b^{\prime} \Rightarrow b=b^{\prime} .
\end{aligned}
$$

The paper of Korner and Olistky [5] shows that the upper bound of $|A||B|$ plays an important role in the zero-error information theory. Cohen gave an upper bound $3^{n}$ for the size of $|A||B|$ on the Boolean lattice while Holzman and Korner improved the bound to $2.3264^{n}$ afterward. Throughout this paper, we study a special class of the recovering pairs $\left(\mathbf{R}_{L}, \mathbf{R}_{L}\right)$ which takes a single set $\mathbf{R}_{L}$. We call $\mathbf{R}_{L}$ a recovering set. As Definition 2.1 and 2.2 shows, a recovering set is a special case of a strongly cancellative set. Here, we focus on the upper bounds and structures of these two sets by using Information Theory.

This paper is organized as follows: Section 2 presents the definitions of strongly cancellative sets, recovering sets, and some results on the entropy function in Information 
Theory. In Section 3, we study the recovering set $\mathbf{R}_{B_{n}}$ on the Boolean lattice $B_{n}$ and find an upper bound $\left|\mathbf{R}_{B_{n}}\right| \leq \sqrt{3} \cdot 2^{0.4056 n}$. As a result, this class of the recovering pairs has an upper bound $3 \cdot 2^{0.8112 n}=3 \cdot(1.7546703)^{n}$ on its size. In Section 4, we study strongly cancellative sets $\mathbf{C}_{B_{n}}$ on $B_{n}$. We give a tight upper bound $2^{\left\lfloor\frac{n}{2}\right\rfloor}$ on $\left|\mathbf{C}_{B_{n}}\right|$ for this lattice. Finally, Section 5 considers the strongly cancellative sets $\mathbf{C}_{D_{l_{1}, \ldots, l_{k}}}$ on the lattice $D_{l_{1}, \ldots, l_{k}}$ which is the product of $k$ chains of length $l_{1}-1, \ldots, l_{k}-1$. We show that when $l_{1}=\cdots=l_{k}=l$, there exists a strongly cancellative set of size $l^{\left\lfloor\frac{k}{2}\right\rfloor}$ and $\left|\mathbf{C}_{D_{l, \ldots, l}}\right| \leq(2 l)^{\frac{k}{2}}+\frac{k(l-1)}{2}+1$.

\section{Preliminaries}

For basic definitions and results concerning lattices, we encourage readers to consult Chapter 3 of [11]. In particular, the Boolean lattice $B_{n}$ is the lattice of all subsets of the set $\{1, \ldots, n\}$ ordered by inclusion, and $D_{l_{1}, \ldots, l_{k}}$ is the lattice formed by the product of $k$ chains of length $l_{1}-1, \ldots, l_{k}-1$, so that the points in $D_{l_{1}, \ldots, l_{k}}$ correspond to $k$-dimensional vectors $\left(v_{1}, \ldots, v_{k}\right)$ with $0 \leq v_{i} \leq l_{i}-1$. The ordering of points in $D_{l_{1}, \ldots, l_{k}}$ is as follows:

$$
v \preceq w \Leftrightarrow v_{i} \leq w_{i}, \text { for all } 1 \leq i \leq k .
$$

A cancellative set is a subset of points in lattice $L$ such that any three different points $v_{1}, v_{2}, v_{3}$ in this set satisfy the following condition:

$$
v_{1} \wedge v_{2} \neq v_{1} \wedge v_{3}
$$

We define strongly cancellative sets as a special class of cancellative sets.

Definition 2.1. A strongly cancellative set $\mathbf{C}_{L}$ of lattice $L$ is a subset of points in $L$ such that for any three different points $a_{1}, a_{2}, a_{3} \in \mathbf{C}_{L}$,

$$
a_{1} \wedge a_{2} \neq a_{1} \wedge a_{3} \text { and } a_{1} \vee a_{2} \neq a_{1} \vee a_{3}
$$

Secondly, a recovering set meets all the conditions that define a strongly cancellative set. In addition, any recovering set $\mathbf{R}_{L}$ forms a recovering pair $\left(\mathbf{R}_{L}, \mathbf{R}_{L}\right)$ on $L$. Here, we give a formal definition for $\mathbf{R}_{L}$.

Definition 2.2. A recovering set $\mathbf{R}_{L}$ of lattice $L$ is a subset of points in $L$ such that for any four different points $a_{1}, a_{2}, a_{3}, a_{4} \in \mathbf{R}_{L}$, we have

$$
\begin{aligned}
& a_{1} \wedge a_{2} \neq a_{3} \wedge a_{4} \text { and } a_{1} \vee a_{2} \neq a_{3} \vee a_{4}, \\
& a_{1} \wedge a_{2} \neq a_{1} \wedge a_{3} \text { and } a_{1} \vee a_{2} \neq a_{1} \vee a_{3} .
\end{aligned}
$$

Now, we introduce the entropy function and show an inequality of it.

Given a discrete random variable $X$ with $m$ possible values $x_{1}, \ldots, x_{m}$, we define the entropy function $\mathcal{H}$ of $X$ as follows:

$$
\mathcal{H}(X)=-\sum_{i=1}^{m} p\left(x_{i}\right) \log _{b} p\left(x_{i}\right)=\sum_{i=1}^{m} p\left(x_{i}\right) \log _{b} \frac{1}{p\left(x_{i}\right)},
$$


where $p$ is the probability mass function of $X$ and $x_{i}$ is the value of $X$. In this paper, we always set $b=2$. Also, the function $x \log \frac{1}{x}$ is concave down when $x>0$. Therefore, for any $s$ values $0 \leq p_{1}, \ldots, p_{s} \leq 1$, we have

$$
\sum_{j=1}^{s}\left(p_{j} \log \frac{1}{p_{j}}\right) \leq s \cdot\left(\frac{\sum_{j=1}^{s} p_{j}}{s}\right) \cdot \log \left(\frac{s}{\sum_{j=1}^{s} p_{j}}\right) .
$$

The following inequality of entropy functions is the major inequality throughout this paper. A proof of the inequality is given in [3].

Theorem 2.3. If $\xi=\left(\xi_{1}, \ldots, \xi_{m}\right)$ is an $n$-dimensional random variable, then

$$
\mathcal{H}(\xi) \leq \sum_{i=1}^{n} \mathcal{H}\left(\xi_{i}\right)
$$

\section{Recovering Set on Boolean lattice $B_{n}$}

In this section, we study recovering sets on Boolean lattices where we use $\cap$ and $\cup$ instead of $\wedge$ and $\vee$. In the following theorem, we give an upper bound for $\left|\mathbf{R}_{B_{n}}\right|$.

Theorem 3.1. For any recovering set $\mathbf{R}_{B_{n}}$, we have $\left|\mathbf{R}_{B_{n}}\right| \leq \sqrt{3} \cdot 2^{0.4056 n}$.

Remark 3.2. In particular, $\left(\mathbf{R}_{B_{n}}, \mathbf{R}_{B_{n}}\right)$ is a special class of recovering pairs on the Boolean lattice, and we give a bound $\left(\sqrt{3} \cdot 2^{0.4056 n}\right)^{2}$ which is significantly better than the cardinalities of a general recovering pair discussed in [1], [9], and [10].

Proof. Let us define a random variable $\xi=a_{i} \cap a_{j}$, where $a_{i}$ and $a_{j}$ are independently chosen according to the uniform distribution on $\mathbf{R}_{B_{n}}$. We wish to show that for any value $a$ in $\xi$, there are at most three ordered pairs $\left(a_{i}, a_{j}\right)$ such that $a=a_{i} \cap a_{j}$. Fixed an ordered pair $\left(a_{t}, a_{s}\right)$ for $\left(a_{i}, a_{j}\right)$, and suppose that there exists another ordered pair $\left(a_{t_{1}}, a_{s_{1}}\right)$ such that $a_{t_{1}} \cap a_{s_{1}}=a_{t} \cap a_{s}=a_{s} \cap a_{t}$. We have the following two cases:

1. $a_{t} \neq a_{s}$. By Definition 2.2, $a_{t_{1}}$ and $a_{s_{1}}$ should be the same element in $B_{n}$, and we have the following possible cases:

(a) $a_{t_{1}}=a_{s_{1}} \notin\left\{a_{t}, a_{s}\right\}$.

In this case, since $a_{t} \cap a_{s}=a_{t_{1}} \cap a_{t_{1}}$, the set $a_{t_{1}}$ is contained in $a_{t}$ and $a_{s}$. It follows that $a_{t_{1}} \cap a_{t}=a_{t_{1}} \cap a_{s}$ which contradicts the second requirement of Definition 2.2.

(b) $a_{t_{1}}=a_{s_{1}} \in\left\{a_{t}, a_{s}\right\}$.

This leaves us exactly one choice for $\left(a_{t_{1}}, a_{s_{1}}\right)$.

2. $a_{t}=a_{s}$. This is the same condition as case (b) in (1). That is to say, one of $a_{t_{1}}$ and $a_{s_{1}}$ must be the set $a_{t}$, and $\left(a_{t_{1}}, a_{s_{1}}\right)$ has only two possible choices. 
Consequently, at most three different ordered pairs obtain the same value in $\xi$. We can give a lower bound on the entropy function of $\xi$ based on this property.

For any $a$ in $\xi$, let $\mathbf{C}(a)=\left\{\left(a_{i}, a_{j}\right): a_{i} \cap a_{j}=a\right.$, and $\left.a_{i}, a_{j} \in \mathbf{R}_{B_{n}}\right\}$ and $\mathcal{P}_{a}=$ $\operatorname{Pr}(\xi=a)=\frac{|\mathbf{C}(a)|}{\left|\mathbf{R}_{B_{n}}\right|^{2}}$. By the above argument, we have $|\mathbf{C}(a)| \leq 3$ and $\mathcal{P}_{a} \leq \frac{3}{\left|\mathbf{R}_{B_{n}}\right|^{2}}$, for any $a$ in $\xi$. Considering the entropy function in (2.4), we obtain the following inequality:

$$
\mathcal{H}(\xi)=\sum_{a \in \xi} \mathcal{P}_{a} \log \frac{1}{\mathcal{P}_{a}} \geq \sum_{a \in \xi} \mathcal{P}_{a} \log \frac{\left|\mathbf{R}_{B_{n}}\right|^{2}}{3}=\log \frac{\left|\mathbf{R}_{B_{n}}\right|^{2}}{3} .
$$

On the other hand, $\xi$ is an $n$-dimensional random variable $\left(\xi_{1}, \ldots, \xi_{n}\right)$, where

$$
\xi_{t}= \begin{cases}1, & t \in a_{i} \cap a_{j} . \\ 0, & t \notin a_{i} \cap a_{j} .\end{cases}
$$

We set $\mathbf{R}_{B_{n}}(t)=\left\{a_{i} \mid a_{i} \in \mathbf{R}_{B_{n}}, t \in a_{i}\right\}$ and $\mathcal{P}_{\mathbf{R}_{B_{n}}}(t)=\frac{\left|\mathbf{R}_{B_{n}}(t)\right|}{\left|\mathbf{R}_{B_{n}}\right|}$, for any $1 \leq t \leq n$. This shows that, $\operatorname{Pr}\left(\xi_{t}=1\right)=\left(\mathcal{P}_{\mathbf{R}_{B_{n}}}(t)\right)^{2}$, for any $t \in\{1, \ldots, n\}$. Let us denote $h(x)$ as $x \log \frac{1}{x}+(1-x) \log \frac{1}{1-x}$. We have by Theorem 2.3 that

$$
\log \frac{\left|\mathbf{R}_{B_{n}}\right|^{2}}{3} \leq \mathcal{H}(\xi) \leq \sum_{t=1}^{n} \mathcal{H}\left(\xi_{t}\right)=\sum_{t=1}^{n}\left[h\left(\mathcal{P}_{\mathbf{R}_{B_{n}}}(t)^{2}\right)\right],
$$

Consider the random variable $\xi^{\prime}=a_{i} \cup a_{j}$. Since entropy functions have the property that $h(x)=h(1-x)$, we similarly get

$$
\log \frac{\left|\mathbf{R}_{B_{n}}\right|^{2}}{3} \leq \sum_{t=1}^{n} h\left(1-\left(1-\left(\mathcal{P}_{\mathbf{R}_{B_{n}}}(t)\right)\right)^{2}\right)=\sum_{t=1}^{n} h\left(\left(1-\left(\mathcal{P}_{\mathbf{R}_{B_{n}}}(t)\right)\right)^{2}\right) .
$$

Now, averaging (3.1) and (3.2), we obtain an upper bound for $\log \frac{\left|\mathbf{R}_{B_{n}}\right|^{2}}{3}$, namely:

$$
\begin{aligned}
\log \frac{\left|\mathbf{R}_{B_{n}}\right|^{2}}{3} & \leq \frac{1}{2} \sum_{t=1}^{n}\left[h\left(\mathcal{P}_{\mathbf{R}_{B_{n}}}(t)^{2}\right)+h\left(\left(1-\mathcal{P}_{\mathbf{R}_{B_{n}}}(t)\right)^{2}\right)\right] \\
& \leq \frac{n}{2}\left[\max _{0 \leq x \leq 1}\left(h\left(x^{2}\right)+h\left((1-x)^{2}\right)\right)\right] \\
& \leq \frac{n}{2}\left[\max _{0 \leq x \leq 1}\left(x \frac{h\left(x^{2}\right)}{x}+(1-x) \frac{h\left((1-x)^{2}\right)}{1-x}\right)\right] .
\end{aligned}
$$

Finally, by the work of D. J. Kleitman, J. Shearer and D. Sturtevant [3], we know that the function $\frac{h\left(x^{2}\right)}{x}$ is concave down, hence, Jensen's inequality gives

$$
\max _{0 \leq x \leq 1}\left(x \frac{h\left(x^{2}\right)}{x}+(1-x) \frac{h\left((1-x)^{2}\right)}{1-x}\right) \leq \max _{0 \leq x \leq 1} \frac{h\left(\left(x^{2}+(1-x)^{2}\right)^{2}\right)}{x^{2}+(1-x)^{2}} .
$$


By some simple calculation, one can see that the function $\frac{h\left(x^{2}\right)}{x}$ is decreasing with $\frac{1}{2} \leq x \leq 1$. Therefore, $2 h\left(\frac{1}{4}\right)=0.8112$ is an upper bound for $\frac{h\left(x^{2}\right)+h\left((1-x)^{2}\right)}{2}$, and we obtain an upper bound for $\left|\mathbf{R}_{B_{n}}\right|$ :

$$
\left|\mathbf{R}_{B_{n}}\right| \leq \sqrt{3} \cdot 2^{0.4056 n}
$$

\section{Strongly Cancellative set on Boolean lattice $B_{n}$}

In this section, we show that the maximal size of $\mathbf{C}_{B_{n}}$ on $B_{n}$, see Definition 2.1, is $2^{\left\lfloor\frac{n}{2}\right\rfloor}$. In addition, this is the tightest bound.

Theorem 4.1. There exists a strongly cancellative set $\mathbf{C}_{B_{n}}$ of size $2^{\left\lfloor\frac{n}{2}\right\rfloor}$ on $B_{n}$.

Proof. We construct $\mathbf{C}_{B_{n}}$ as follows. First, let us divide the set $\left\{1, \ldots, 2\left\lfloor\frac{n}{2}\right\rfloor\right\}$ into $\left\lfloor\frac{n}{2}\right\rfloor$ blocks $S_{i}=\{2 i-1,2 i\}$, for $1 \leq i \leq\left\lfloor\frac{n}{2}\right\rfloor$. We define $\mathbf{C}_{B_{n}}$ to be the family of all the subsets $s=\left\{s_{1}, \ldots, s_{\left\lfloor\frac{n}{2}\right\rfloor}\right\}$ such that $s_{i} \in S_{i}$, for $1 \leq i \leq\left\lfloor\frac{n}{2}\right\rfloor$. Thus, we have $\left|\mathbf{C}_{B_{n}}\right|=2^{\left\lfloor\frac{n}{2}\right\rfloor}$. Now, we show that $\mathbf{C}_{B_{n}}$ satisfies the conditions defining strongly cancellative set.

Consider different elements $b=\left\{b_{1}, \ldots, b_{\left\lfloor\frac{n}{2}\right\rfloor}\right\}$ and $c=\left\{c_{1}, \ldots, c_{\left\lfloor\frac{n}{2}\right\rfloor}\right\}$ in $\mathbf{C}_{B_{n}}$, so that there exists some $1 \leq k \leq\left\lfloor\frac{n}{2}\right\rfloor$ such that $b_{k} \neq c_{k}$. Without lost of generality, assume that $b_{k}=2 k-1$ and $c_{k}=2 k$. Consequently, for any element $a=\left\{a_{1}, \ldots, a_{\left\lfloor\frac{n}{2}\right\rfloor}\right\}$, we have the following properties:

1. $b_{k} \notin a \cap c$ and $c_{k} \notin a \cap b$,

2. $b_{k} \in a \cup b$ and $c_{k} \in a \cup c$,

3. $a_{k}=b_{k}$ or $a_{k}=c_{k}$,

4. $b_{k} \in a \cap b$ or $c_{k} \in a \cap c$,

5. $c_{k} \notin a \cup b$ or $b_{k} \notin a \cup c$.

Clearly, property (3) implies (4) and (5). Moreover, (1) and (4) imply that $a \cap b \neq a \cap c$, and similarly, (2) and (5) imply that $a \cup b \neq a \cup c$. Therefore, $\mathbf{C}_{B_{n}}$ is a strongly cancellative set.

Now, we show that $\left|\mathbf{C}_{B_{n}}\right| \leq 2^{\left\lfloor\frac{n}{2}\right\rfloor}$.

Theorem 4.2. For any strongly cancellative $\mathbf{C}_{B_{n}}$ on $B_{n}$, we have $\left|\mathbf{C}_{B_{n}}\right| \leq 2^{\left\lfloor\frac{n}{2}\right\rfloor}$. 
Proof. Fix an element $v^{\prime} \in \mathbf{C}_{B_{n}}$. We consider the following sets:

$$
\begin{aligned}
& \mathbf{C}_{1}\left(v^{\prime}\right)=\left\{v \cap v^{\prime}: v \neq v^{\prime}, v \in \mathbf{C}_{B_{n}}\right\}, \\
& \mathbf{C}_{2}\left(v^{\prime}\right)=\left\{v \cup v^{\prime}: v \neq v^{\prime}, v \in \mathbf{C}_{B_{n}}\right\} .
\end{aligned}
$$

By Equation (2.1), we have $\left|\mathbf{C}_{1}\left(v^{\prime}\right)\right|=\left|\mathbf{C}_{2}\left(v^{\prime}\right)\right|=\left|\mathbf{C}_{B_{n}}\right|-1$. This implies that

$$
\left|\mathbf{C}_{B_{n}}\right| \leq 1+\min \left(\left|\left\{v: v \subseteq v^{\prime}\right\}\right|,\left|\left\{v: v \supseteq v^{\prime}\right\}\right|\right) .
$$

Moreover,

$$
\min \left(\left|\left\{v: v \subseteq v^{\prime}\right\}\right|,\left|\left\{v: v \supseteq v^{\prime}\right\}\right|\right) \leq\left|\left\{v: v \subseteq v^{*}, \operatorname{rank}\left(v^{*}\right)=\left\lfloor\frac{n}{2}\right\rfloor\right\}\right| .
$$

We consider the following two cases:

1. $2 \mid n$. Then we have $\operatorname{rank}\left(v^{\prime}\right)=\left\lfloor\frac{n}{2}\right\rfloor$ if the equality holds in (4.2). Suppose that the equalities in (4.1) and (4.2) hold for every $v^{\prime} \in \mathbf{C}_{B_{n}}$. Consequently, $\operatorname{rank}\left(v^{\prime}\right)=\left\lfloor\frac{n}{2}\right\rfloor$, for every $v^{\prime} \in \mathbf{C}_{B_{n}}$, which implies that any two elements in the set are incomparable. One can easily see that, $\mathbf{C}_{1}\left(v^{\prime}\right) \neq\left|\left\{v: v \subseteq v^{\prime}\right\}\right|$ and $\mathbf{C}_{2}\left(v^{\prime}\right) \neq\left|\left\{v: v \supseteq v^{\prime}\right\}\right|$. Therefore, the equalities in (4.1) and (4.2) can not hold at the same time.

2. $2 \nmid n$. Then $\operatorname{rank}\left(v^{\prime}\right)=\left\lfloor\frac{n}{2}\right\rfloor$ or $\left\lfloor\frac{n+1}{2}\right\rfloor$ if the equality hold in (4.2). Suppose that the equalities in (4.1) and (4.2) holds for every $v^{\prime} \in \mathbf{C}_{B_{n}}$. Pick some element $w \in \mathbf{C}_{B_{n}}$. If $\operatorname{rank}(w)=\left\lfloor\frac{n}{2}\right\rfloor$, then by (4.1) there exist other two elements $w^{\prime}$ and $w^{\prime \prime}$ in the set such that $w \cap w^{\prime}=w$ and $w \cap w^{\prime \prime}=\emptyset$. This implies that $\operatorname{rank}\left(w^{\prime}\right)=\left\lfloor\frac{n+1}{2}\right\rfloor$ and $w^{\prime} \backslash w=\{x\}$, where $1 \leq x \leq n$.

By Equation (2.1), we have $\emptyset=w \cap w^{\prime \prime} \neq w^{\prime} \cap w^{\prime \prime}$, and thus $x \in w^{\prime \prime}$. This means that $w \cup w^{\prime \prime}=w^{\prime} \cup w^{\prime \prime}$ which is not possible. As a result, the equalities in (4.1) and (4.2) cannot hold at the same time. Similarly, one can prove the same statement when $\operatorname{rank}(w)=\left\lfloor\frac{n+1}{2}\right\rfloor$.

Finally, from (1) and (2), we have

$$
\left|\mathbf{C}_{B_{n}}\right| \leq\left|\left\{v: v \subseteq v^{*}, \operatorname{rank}\left(v^{*}\right)=\left\lfloor\frac{n}{2}\right\rfloor\right\}\right|=2^{\left\lfloor\frac{n}{2}\right\rfloor} .
$$

\section{Strongly Cancellative Sets on lattices $D_{l_{1}, \ldots, l_{k}}$ and $D_{l}^{k}$}

For the definition of the lattice $D_{l_{1}, \ldots, l_{k}}$, see Section 2. In particular, we say that $D_{l}^{k}$ is a lattice of $k$ chains of length $l-1$. It is easy to show that for any two points $v=\left(v_{1}, \ldots, v_{k}\right)$ and $v^{\prime}=\left(v_{1}^{\prime}, \ldots, v_{k}^{\prime}\right)$ in $D_{l_{1}, \ldots, l_{k}}$,

$$
\begin{aligned}
& \left(v_{1}, \ldots, v_{k}\right) \wedge\left(v_{1}^{\prime}, \ldots, v_{k}^{\prime}\right)=\left(\min \left(v_{1}, v_{1}^{\prime}\right), \ldots, \min \left(v_{k}, v_{k}^{\prime}\right)\right), \\
& \left(v_{1}, \ldots, v_{k}\right) \vee\left(v_{1}^{\prime}, \ldots, v_{k}^{\prime}\right)=\left(\max \left(v_{1}, v_{1}^{\prime}\right), \ldots, \max \left(v_{k}, v_{k}^{\prime}\right)\right) .
\end{aligned}
$$


In the following proposition, we give a tight bound for the size of strongly cancellative sets on $D_{l_{1}, l_{2}}$.

Proposition 5.1. Let $\mathbf{C}_{D_{l_{1}, l_{2}}}$ be a strongly cancellative set on the lattice $D_{l_{1}, l_{2}}$. Then

$$
\left|\mathbf{C}_{D_{l_{1}, l_{2}}}\right| \leq \min \left(l_{1}, l_{2}\right)
$$

Proof. Without lost of generality, we assume that $l_{1} \leq l_{2}$. Every point $v$ in $D_{l_{1}, l_{2}}$ is a vector $\left(v_{1}, v_{2}\right)$, where $0 \leq v_{1} \leq l_{1}-1$ and $0 \leq v_{2} \leq l_{2}-1$. We proceed by contradiction.

Suppose that $\left|\mathbf{C}_{D_{l_{1}, l_{2}}}\right|>l_{1}$. Then there exists two points $v=\left(v_{1}, v_{2}\right)$ and $w=\left(w_{1}, w_{2}\right)$ such that $v_{1}=w_{1}$ and $v_{2}<w_{2}$. For any point $v^{*}=\left(v_{1}^{*}, v_{2}^{*}\right) \notin\{v, w\}$, all the following four possible cases lead to contradiction:

1. $v_{2}^{*} \leq v_{2}$ implies that $v^{*} \wedge v=v^{*} \wedge w$.

2. $v_{2}^{*}>w_{2}$ implies that $v^{*} \vee v=v^{*} \vee w$.

3. $v_{2} \leq v_{2}^{*} \leq w_{2}$ and $v_{1}^{*} \leq v_{1}$ imply that $v^{*} \vee w=v \vee w$.

4. $v_{2} \leq v_{2}^{*} \leq w_{2}$ and $v_{1}^{*} \geq v_{1}$ imply that $v^{*} \wedge v=v \wedge w$.

Therefore, we must have $\left|\mathbf{C}_{D_{l_{1}, l_{2}}}\right| \leq l_{1}=\min \left(l_{1}, l_{2}\right)$, as desired.

The bound $\min \left(l_{1}, l_{2}\right)$ is tight for $\left|\mathbf{C}_{D_{l_{1}, l_{2}}}\right|$. In particular, it is not hard to show that the following set is a strongly cancellative set of size $\min \left(l_{1}, l_{2}\right)$ :

$$
\mathbf{C}_{D_{l_{1}, l_{2}}}=\left\{(x, y) \mid x+y=\min \left(l_{1}, l_{2}\right)-1\right\} \text {. }
$$

In the following, we study the size of the strongly cancellative sets on $D_{l}^{k}$.

Proposition 5.2. Suppose that $\mathbf{C}_{k_{1}}$ is a strongly cancellative set on the lattice $D_{l}^{k_{1}}$ for some small $k_{1}$, and any two elements in $\mathbf{C}_{k_{1}}$ are incomparable. Then, for any $k$ with $\left\lfloor\frac{k}{k_{1}}\right\rfloor=s$, there is a strongly cancellative set $\mathbf{C}_{k}$ of size $\left|\mathbf{C}_{k_{1}}\right|^{s}$ on $D_{l}^{k}$.

Proof. Every point in $D_{l}^{k}$ is a $k$-dimensional vector $\left(v_{1}, \ldots, v_{k}\right)$, where $0 \leq v_{i} \leq l-1$ for $1 \leq i \leq k$. For every vector $v=\left(v_{1}, \ldots, v_{k}\right)$, we define subvectors induced by $v$ as $B_{j}(v)=\left(v_{(j-1) k_{1}+1}, \ldots, v_{j k_{1}}\right)$, for $1 \leq j \leq s$, and $B_{s+1}(v)=\left(v_{k_{1}+1}, \ldots, v_{k}\right)$. Let $\mathbf{C}_{k}$ to be the set of all $k$-dimensional vectors $v$ such that $B_{j}(v) \in \mathbf{C}_{k_{1}}$ for all $1 \leq j \leq s$, and $B_{s+1}(v)$ is the zero vector. Clearly, we have $\left|\mathbf{C}_{k}\right|=\left|\mathbf{C}_{k_{1}}\right|^{s}$.

Suppose there are three different elements $v, v^{\prime}, v^{\prime \prime} \in \mathbf{C}_{k}$ such that $v \vee v^{\prime}=v \vee v^{\prime \prime}$. Since $v^{\prime}$ and $v^{\prime \prime}$ are different, we have $B_{j^{*}}\left(v^{\prime}\right) \neq B_{j^{*}}\left(v^{\prime \prime}\right)$ for some $1 \leq j^{*} \leq s$. On the other hand, we know $B_{j^{*}}(v) \vee B_{j^{*}}\left(v^{\prime}\right)=B_{j^{*}}(v) \vee B_{j^{*}}\left(v^{\prime \prime}\right)$ which implies that one of $B_{j^{*}}\left(v^{\prime}\right)$ or $B_{j^{*}}\left(v^{\prime \prime}\right)$ is equal to $B_{j^{*}}(v)$. Therefore, $v_{i}^{\prime} \preceq v_{i}^{\prime \prime}$ or $v_{i}^{\prime \prime} \preceq v_{i}^{\prime}$, and this contradicts the assumption that any two different elements in $\mathbf{C}_{k_{1}}$ are incomparable. Similarly, it is easy to see that $v \wedge v^{\prime} \neq v \wedge v^{\prime \prime}$. As a result, $\mathbf{C}_{k}$ is a strongly cancellative set of size $\left|\mathbf{C}_{k_{1}}\right|^{s}$. 
We can use this result to give a construction of a strongly cancellative set on $D_{l}^{k}$.

Corollary 5.3. There exists a strongly cancellative set $\mathbf{C}_{D_{l}^{k}}$ on the lattice $D_{l}^{k}$, such that $\left|\mathbf{C}_{D_{l}^{k}}\right|=l^{\left\lfloor\frac{k}{2}\right\rfloor}$.

Proof. We have seen that $\mathbf{C}_{D_{l}^{2}}=\{(x, y) \mid x+y=l-1\}$ is a strongly cancellative set of size $l$ on $D_{l}^{2}$ such that any two elements in the set are incomparable. By Proposition 5.2, there exists a strongly cancellative set $\mathbf{C}_{D_{l}^{k}}$ of size $l^{\left\lfloor\frac{k}{2}\right\rfloor}$. $D_{l}^{k}$.

We end this section with an upper bound of the size of strongly cancellative sets on

Theorem 5.4. Let $\mathbf{C}_{D_{l}^{k}}$ be a strongly cancellative set on $D_{l}^{k}$, then

$$
\left|\mathbf{C}_{D_{l}^{k}}\right| \leq(2 l)^{\frac{k}{2}}+\frac{k(l-1)}{2}+1 .
$$

Proof. Any element $v$ on the lattice $D_{l}^{k}$ is a $k$-dimensional vector $v=\left(v_{1}, \ldots, v_{k}\right)$ such that $0 \leq v_{i} \leq l-1$ for all $1 \leq i \leq k$. We first define $\mathbf{C}_{m}(t)$ and $\mathcal{P}_{m}(t)$.

1. We define $\mathbf{C}_{m}(t)$ to be set of vectors whose $m$-th component is $t$, for any $1 \leq t \leq k$. That is, $\mathbf{C}_{m}(t)=\left\{v \mid v \in \mathbf{C}_{D_{l}^{k}}, v_{m}=t\right\}$.

2. Let $v$ be a random element uniformly chosen in the set $\mathbf{C}_{D_{l}^{k}}$. We denote the probability that the $m$-th component $v_{m}$ of $v$ is $t$ by $\mathcal{P}_{m}(t)$. So,

$$
\mathcal{P}_{m}(t)=\frac{\left|\mathbf{C}_{m}(t)\right|}{\left|\mathbf{C}_{D_{l}^{k}}\right|} .
$$

Fix an arbitrary element $v \in \mathbf{C}_{D_{l}^{k}}$. We define the random variable $\xi_{v}=v \wedge v^{*}$, where $v^{*}$ is the random element uniformly chosen in $\mathbf{C}_{D_{l}^{k}} \backslash\{v\}$. Suppose that there exist two elements $v_{1}$ and $v_{2}$ in $\mathbf{C}_{D_{l}^{k}}$ so that we obtain the same value in $\xi_{v}$. That is, $v \wedge v_{1}=v \wedge v_{2}$ which is not possible in strongly cancellative sets. Consequently, every value in $\xi_{v}$ appears exactly once. Since there are totally $\left|\mathbf{C}_{D_{l}^{k}}\right|-1$ different values for $\xi_{v}$, the entropy function of $\xi_{v}$ is

$$
\mathcal{H}\left(\xi_{v}\right)=\log \left(\left|\mathbf{C}_{D_{l}^{k}}\right|-1\right) .
$$

For convenience, we set $N=\left|\mathbf{C}_{D_{l}^{k}}\right|-1$.

On the other hand, every value in $\xi_{v}$ is a $k$-dimensional vector $\left(\xi_{v}(1), \ldots, \xi_{v}(k)\right)$ such that $\xi_{v}(m)=\min \left(v_{m}, v_{m}^{*}\right)$ for any $1 \leq m \leq k$ and randomly chosen element $v^{*}$. Consequently, for any $1 \leq m \leq k, \xi_{v}(m)$ takes all its values in $\left\{0,1, \ldots, v_{m}\right\}$. We denote the probability that $\xi_{v}(m)=t^{\prime}$ by $\mathcal{P}_{\xi_{v}(m)}\left(t^{\prime}\right)$. Moreover, if $0 \leq t^{\prime} \leq v_{m}-1$, we should have $t^{\prime}=\min \left(v_{m}, v_{m}^{*}\right)<v_{m}$ and thus, $v_{m}^{*}=t^{\prime}$. If $t^{\prime}=v_{m}$, we must have $\min \left(v_{m}, v_{m}^{*}\right)=t^{\prime}=v_{m}$ which implies that $v_{m} \leq v_{m}^{*}$. 
Therefore, we obtain the following properties for $P_{\xi_{v}(m)}\left(t^{\prime}\right)$ :

$$
\mathcal{P}_{\xi_{v}(m)}\left(t^{\prime}\right)=\left\{\begin{aligned}
\frac{\left|\mathbf{C}_{m}\left(t^{\prime}\right)\right|}{N}, & 0 \leq t^{\prime} \leq v_{m}-1 . \\
\frac{\left[\sum_{t_{1}^{\prime}=v_{m}}^{l-1}\left|\mathbf{C}_{m}\left(t_{1}^{\prime}\right)\right|\right]-1}{N}, & t^{\prime}=v_{m} . \\
0, & v_{m}+1 \leq t^{\prime} \leq l-1 .
\end{aligned}\right.
$$

The entropy function of $\xi_{v}(m)$ can be computed as follows:

$$
\begin{aligned}
\mathcal{H}\left(\xi_{v}(m)\right) & =\mathcal{H}\left(\mathcal{P}_{\xi_{v}(m)}(0), \ldots, \mathcal{P}_{\xi_{v}(m)}\left(v_{m}-1\right), \mathcal{P}_{\xi_{v}(m)}\left(v_{m}\right)\right) \\
& =\sum_{t^{\prime}=0}^{v_{m}} \mathcal{P}_{\xi_{v}(m)}\left(t^{\prime}\right) \log \frac{1}{\mathcal{P}_{\xi_{v}(m)}\left(t^{\prime}\right)} .
\end{aligned}
$$

Furthermore, by Eq.(5.1) and Theorem (2.3), we have

$$
\log N \leq \sum_{m=1}^{k} \mathcal{H}\left(\xi_{v}(m)\right)=\sum_{m=1}^{k} \sum_{t^{\prime}=0}^{v_{m}} \mathcal{P}_{\xi_{v}(m)}\left(t^{\prime}\right) \log \frac{1}{\mathcal{P}_{\xi_{v}(m)}\left(t^{\prime}\right)} .
$$

The above equation holds for every element $v$ in the set $\mathbf{C}_{D_{l}^{k}}$. If we take the average over all the elements in the set $\mathbf{C}_{D_{l}^{k}}$, we obtain

$$
\log N \leq \frac{\sum_{v \in \mathbf{C}_{D_{l}^{k}}} \sum_{m=1}^{k} \mathcal{H}\left(\xi_{v}(m)\right)}{N+1}=\frac{\sum_{m=1}^{k} \sum_{v \in \mathbf{C}_{D_{l}^{k}}} \mathcal{H}\left(\xi_{v}(m)\right)}{N+1}
$$

Moreover, from (2), we know that the probability that $v_{m}=t$ for some $0 \leq t \leq l-1$ is $\mathcal{P}_{m}(t)=\frac{\left|\mathbf{C}_{m}(t)\right|}{N+1}$, and therefore, (5.4) can be rewritten as follows:

$$
\log N \leq \sum_{m=1}^{k} \sum_{t=0}^{l-1} \mathcal{P}_{m}(t)\left(\sum_{t^{\prime}=0}^{t} P_{\xi_{v}(m)}\left(t^{\prime}\right) \log \frac{1}{\mathcal{P}_{\xi_{v}(m)}\left(t^{\prime}\right)}\right) .
$$

Now, we consider the random variable $\xi_{v}^{\prime}=v \vee v^{*}$, where $v^{*}$ is also independently chosen under the uniform distribution on $\mathbf{C}_{D_{l}^{k}} \backslash\{v\}$. Thus, we have the following:

$$
\mathcal{P}_{\xi_{v}^{\prime}(m)}\left(t^{\prime}\right)=\left\{\begin{aligned}
0, & 0 \leq t^{\prime} \leq v_{m}-1 . \\
\frac{\left[\sum_{t_{1}^{\prime}=0}^{v_{m}}\left|\mathbf{C}_{m}\left(t_{1}^{\prime}\right)\right|\right]-1}{N}, & t^{\prime}=v_{m} . \\
\frac{\left|\mathbf{C}_{m}\left(t^{\prime}\right)\right|}{N}, & v_{m}+1 \leq t^{\prime} \leq l-1 .
\end{aligned}\right.
$$

By similar arguments, Eq.(5.6) implies that:

$$
\log N \leq \sum_{m=1}^{k} \sum_{t=0}^{l-1} \mathcal{P}_{m}(t)\left(\sum_{t^{\prime}=t}^{l-1} \mathcal{P}_{\xi_{v}(m)}\left(t^{\prime}\right) \log \frac{1}{\mathcal{P}_{\xi_{v}(m)}\left(t^{\prime}\right)}\right) .
$$


For convenience, let $\mathcal{P}_{m}^{\prime}\left(t^{\prime}\right)=\frac{\left|\mathbf{C}_{m}\left(t^{\prime}\right)\right|}{N}$. Also, we set $q_{m}(t)=\frac{\left[\sum_{t_{1}^{\prime}=t}^{l-1}\left|\mathbf{C}_{m}\left(t_{1}^{\prime}\right)\right|\right]-1}{N}$, and $q_{m}^{\prime}(t)=$ $\frac{\left[\sum_{t_{1}^{\prime}=0}^{t}\left|\mathbf{C}_{m}\left(t_{1}^{\prime}\right)\right|\right]-1}{N}$.

Consider the following inequality,

$$
\begin{aligned}
& \sum_{t^{\prime}=0}^{t} \mathcal{P}_{\xi_{v}(m)}\left(t^{\prime}\right) \log \frac{1}{\mathcal{P}_{\xi_{v}(m)}\left(t^{\prime}\right)}+\sum_{t^{\prime}=t}^{l-1} \mathcal{P}_{\xi_{v}(m)}\left(t^{\prime}\right) \log \frac{1}{\mathcal{P}_{\xi_{v}(m)}\left(t^{\prime}\right)} \\
\leq & \left(\sum_{t^{\prime}=0}^{l-1} \mathcal{P}_{m}^{\prime}\left(t^{\prime}\right) \log \frac{1}{\mathcal{P}_{m}^{\prime}\left(t^{\prime}\right)}\right)+q_{m}(t) \log \frac{1}{q_{m}(t)}+q_{m}^{\prime}(t) \log \frac{1}{q_{m}^{\prime}(t)} \\
\leq & \left(\frac{N+1}{N}\right) \log \frac{l N}{N+1}+\left(q_{m}(t)+q_{m}^{\prime}(t)\right) \cdot \log \left(\frac{2}{q_{m}(t)+q_{m}^{\prime}(t)}\right) .
\end{aligned}
$$

Note that (5.9) holds because $p \log \frac{1}{p}>0$, when $0<p<1$, and (5.10) holds by the inequality in (2.5).

Finally, by adding (5.5) and (5.7), the above result implies that

$$
\begin{aligned}
2 \log N & \leq \sum_{m=1}^{k} \sum_{t=0}^{l-1} \mathcal{P}_{m}(t)\left[\left(q_{m}(t)+q_{m}^{\prime}(t)\right) \cdot \log \left(\frac{2}{q_{m}(t)+q_{m}^{\prime}(t)}\right)+\left(1+\frac{1}{N}\right) \log l\right] \\
& =k\left(1+\frac{1}{N}\right) \log l+\sum_{m=1}^{k} \sum_{t=0}^{l-1} \mathcal{P}_{m}(t) \cdot\left(q_{m}(t)+q_{m}^{\prime}(t)\right) \cdot \log \left(\frac{2}{q_{m}(t)+q_{m}^{\prime}(t)}\right) \\
& \leq k+k\left(1+\frac{1}{N}\right) \log l
\end{aligned}
$$

The last inequality is due to the fact that function $x \log \frac{2}{x}$ is decreasing with $x \geq 1$ and that $q_{m}(t)+q_{m}^{\prime}(t)=1+\frac{\left|\mathbf{C}_{m}(t)\right|-1}{N} \geq 1$ when $\mathcal{P}_{m}(t)=\frac{\left|\mathbf{C}_{m}(t)\right|}{N+1} \neq 0$.

Therefore, we have

$$
N \leq 2^{\frac{k}{2}} l^{\frac{k}{2}\left(1+\frac{1}{N}\right)} .
$$

Consider the function $f(N)=N-2^{\frac{k}{2}} l^{\frac{k}{2}\left(1+\frac{1}{N}\right)}$. The inequality (5.11) implies that $f(N) \leq 0$ and is increasing with $N$. If we set $N_{1}=(2 l)^{\frac{k}{2}}+\frac{k(l-1)}{2}$, then it is easy to see that

$$
\begin{aligned}
f\left(N_{1}\right) & =\frac{k(l-1)}{2}+(2 l)^{\frac{k}{2}} \cdot\left(1-(1+l-1)^{\frac{k}{2 N_{1}}}\right) \\
& \geq \frac{k(l-1)}{2}+(2 l)^{\frac{k}{2}} \cdot\left(1-\left(1+\frac{(l-1) k}{2 N_{1}}\right)\right) \\
& =\frac{k(l-1)}{2}-\frac{(2 l)^{\frac{k}{2}}}{N_{1}} \cdot \frac{k(l-1)}{2} \geq 0,
\end{aligned}
$$

where (5.12) implies (5.13) because $(1+a)^{b} \leq 1+a b$ when $b \leq 1$ and $a \geq 0$. 
As a result, since $f(N) \leq 0 \leq f\left(N_{1}\right)$,

$$
\left|\mathbf{C}_{D_{l}^{k}}\right|-1=N \leq N_{1}=(2 l)^{\frac{k}{2}}+\frac{k(l-1)}{2} .
$$

\section{Acknowledgement}

Part of this research was done at MIT SPUR program. We would like to thank Clifford Smyth for proposing this problem.

\section{References}

[1] A. Sali, G. Simonyi: Recovering Set System and Sandglass Conjecture, Combinatorics, Probability, and Computing (1997) 6, 481-491

[2] CsÁkÁny Rita: Some results on the sandglass conjecture. (English summary) 6th International Conference on Graph Theory (Marseille, 2000), 4 pp. (electronic), Electron. Notes Discrete Math., 5, Elsevier, Amsterdam, 2000.

[3] D. J. Kleitman, J. Shearer and D. Sturtevant: Intersections of k -element sets, vol. 1, Combinatorica, 1981.

[4] I. CsiszÁr, J. Konner: Information Theory: Coding Theorems for Discrete Memoryless Systems, Academic Press, New York, 1982.

[5] Janos Korner, Alon Orlitsky: Zero-Error Information Theory, Ieee Transactions On Information Thory, vol. 44, no. 6, October 1998.

[6] James B. Shearer: A New Construction For Cancellative Families of Sets, The Electronic Journal of Combinatorics 3 (1996).

[7] Michael L. Fredman: The Complexity of Maintaining an Array and Computing Its Partial Sums, Journal of the Association for Computing Machinery, Vol. 29, no I, January 1982, pp 250-260

[8] P. FrankL, Z. Füredi, Union-free Hypergraphs and Probability Theory, European Journal of Combinatorics, 5 (1984), pp. 127-131.

[9] R. Ahlswede, G. Simonyi: On the optimal structure of recovering set pairs in lattices: the sandglass conjecture, Discr. Math., 128(1994), 389-394

[10] R. Holzman, J. Korner: Cancellative Pairs of Families of Sets, Europ. J. Combinatorics (1995) 16, 263-266.

[11] R. Stanley: Enumerative Combinatorics, vol. 1, Cambridge University Press, New York/Cambridge, 1999. 